\title{
Editorial
}

\section{Big Data Processing and Modeling in Solar Physics}

\author{
X. Huang $\mathbb{D}^{1}{ }^{1}$ I. Usoskin, ${ }^{2}$ L. Y. Zhang, ${ }^{2}$ and H. N. Wang ${ }^{1}$ \\ ${ }^{1}$ Key Laboratory of Solar Activity, National Astronomical Observatories of Chinese Academy of Sciences, Beijing, China \\ ${ }^{2}$ Space Physics Unit and Sodankylä Geophysical Observatory, University of Oulu, Oulu, Finland
}

Correspondence should be addressed to X. Huang; xhuang@bao.ac.cn

Received 25 February 2020; Accepted 25 February 2020; Published 17 March 2020

Copyright $\odot 2020$ X. Huang et al. This is an open access article distributed under the Creative Commons Attribution License, which permits unrestricted use, distribution, and reproduction in any medium, provided the original work is properly cited.

The Sun is the energy source of the Earth. The electromagnetic environment of the Earth is affected by solar activity, and the impact of violent activity bursts can reach the Earth within eight minutes. Hence the detection, recognition, and prediction of solar activity are essential.

The physical mechanisms of solar activity bursts are not yet completely clear. However, a large number of data have been accumulated and solar observation instruments can record the multiwavelength imaging data every day with high cadence. In order to cope with the rapidly growing amount of solar data, there is an increasing need for automatic detection and prediction technologies.

This special issue is focused on solar data mining technology. We invited authors to contribute with original research articles in this special issue. Eleven original research manuscripts have been received. After the peer-reviewed process, seven of them were accepted for publications. Therein, three papers focused on the detection and recognition of regions of interest in the solar images, two papers presented research on the short-term and midterm solar activity prediction, respectively, and one paper discussed the influence of solar activity on economic activities. From these articles, we can find that the machine learning methods, especially the deep learning methods, play an important role in solar activity monitoring and prediction. Finally, we hope that researchers will find this special issue useful.

\section{Conflicts of Interest}

The editors declare that they have no conflicts of interest regarding the publication of this special issue.

\section{Acknowledgments}

We sincerely thank the authors contributing to the special issue and referees thoroughly reviewing the papers. The special issue also benefits from the members of the editorial board of Advances in Astronomy.

X. Huang

I. Usoskin

L. Y. Zhang

H. N. Wang 